\title{
Non-Hermitian Spectra and Anderson Localization
}

\author{
Luca G. Molinari \\ Dipartimento di Fisica and I.N.F.N. sezione di Milano \\ Via Celoria 16, 20133 Milano, Italy \\ E-mail: luca.molinari@mi.infn.it
}

(Dated: october 2008)

\begin{abstract}
The spectrum of exponents of the transfer matrix provides the localization lengths of Anderson's model for a particle in a lattice with disordered potential. I show that a duality identity for determinants and Jensen's identity for subharmonic functions, give a formula for the spectrum in terms of eigenvalues of the Hamiltonian with non-Hermitian boundary conditions. The formula is exact; it involves an average over a Bloch phase, rather than disorder. A preliminary investigation of non-Hermitian spectra of Anderson's model in $\mathrm{D}=1,2$ and on the smallest exponent is presented.
\end{abstract}

PACS numbers: 71.23.An (theories and models, localized states), 02.20.-a (Matrix theory)

\section{INTRODUCTION}

Several models in physics are described by matrices with banded or block-tridiagonal structure. Examples are the Laplacian matrix, the Anderson Hamiltonian for transport in a lattice with random impurities, band random matrices, tight binding models in condensed matter and chemistry. The matrix structure reproduces that of a system consisting of a chain of units with same number of internal states, with nearest neighbors interaction. Finite size effects are often dealt with by imposing periodicity; the limit of large number of units is eventually taken.

The matrix structure calls for a transfer matrix description of the eigenstates, and the spectrum of exponents of the transfer matrix describes the decay lengths of the eigenstates. For the Anderson model or band random matrices, most of the knowledge on Lyapunov spectra relies on numerical computations.

With great generality, I showed that an analytic tool to access the decay lengths is a duality relation, that connects the spectrum of a non-Hermitian extension of the block-tridiagonal matrix, with the spectrum of the related transfer matrix ${ }^{1,2,3}, 4$. The extension arises by mere generalization of boundary conditions for the eigenstates. If $\vec{u}_{k}$ specifies the state of a unit of the chain $(k=1, \ldots, n)$, the boundary conditions (b.c.) are parametrized by a complex number $z$ :

$$
\vec{u}_{n+1}=z^{n} \vec{u}_{1}, \quad \vec{u}_{0}=\frac{1}{z^{n}} \vec{u}_{n}
$$

This destroys Hermiticity of the Hamiltonian matrix, but enlights a nice property of the transfer matrix:

$$
T(\epsilon)\left[\begin{array}{c}
\vec{u}_{1} \\
\frac{1}{z^{n}} \vec{u}_{n}
\end{array}\right]=z^{n}\left[\begin{array}{c}
\vec{u}_{1} \\
\frac{1}{z^{n}} \vec{u}_{n}
\end{array}\right]
$$

The ensuing spectral duality and Jensen's identity for subharmonic functions allow to evaluate the counting function of exponents. This paper is intended to introduce the theory and explore its application to the longstudied problem of Anderson's localization. In section
II the duality relation is reviewed and the main formula (12) for the exponents is obtained from Jensen's theorem. The theory can be extended to include the spectrum of the (Lyapunov) exponents of the matrix $\mathrm{T}^{\dagger} \mathrm{T}$, by constructing a corresponding non-Hermitian block tridiagonal matrix, twice the size of the original Hamiltonian matrix. In section III a preliminary study of the eigenvalues of non Hermitian Hamiltonian matrices in $\mathrm{D}=1$ and $\mathrm{D}=2$ is made, with the purpose of illustrating the duality. The spectral formula is used to evaluate the smallest exponent $\xi_{\min }$, in a regime where the eigenvalues are already all complex.

Because of their relevance in mathematics, numerical analysis and physics, block tridiagonal matrices are an active area of research $5,6,7$. This work extends in a new perspective the work by Hatano and Nelson ${ }^{8}$ which, together with the works by Feinberg and Zee ${ }^{\underline{9}}$, started an interest for non-Hermitian matrix models in physics.

\section{THEORY}

\section{A. Transfer Matrix}

Consider the following block tridiagonal matrix with corners, of size $n m \times n m$,

$$
H=\left[\begin{array}{cccc}
A_{1} & B_{1} & & B_{n}^{\dagger} \\
B_{1}^{\dagger} & \ddots & \ddots & \\
& \ddots & \ddots & B_{n-1} \\
B_{n} & & B_{n-1}^{\dagger} & A_{n}
\end{array}\right]
$$

The blocks have size $m \times m: B_{k}$ are complex matrices with $\operatorname{det} B_{k} \neq 0, A_{k}$ are Hermitian matrices. To the matrix $\epsilon I_{n m}-H$ there corresponds the transfer matrix 1

$$
\begin{aligned}
& T(\epsilon)=\left[\begin{array}{cc}
B_{n}^{-1}\left(\epsilon I_{m}-A_{n}\right) & -B_{n}^{-1} B_{n-1}^{\dagger} \\
I_{m} & 0
\end{array}\right] \times \\
& \cdots \times\left[\begin{array}{cc}
B_{1}^{-1}\left(\epsilon I_{m}-A_{1}\right) & -B_{1}^{-1} B_{n}^{\dagger} \\
I_{m} & 0
\end{array}\right] .
\end{aligned}
$$


$I_{m}$ is the $m \times m$ identity matrix. The transfer matrix is so named because it transforms the eigenvalue equation $H u=\epsilon u$ into a relation for the end-components of the vector $u=\left(\vec{u}_{1}, \ldots, \vec{u}_{n}\right)^{t}$ :

$$
T(\epsilon)\left[\begin{array}{c}
\vec{u}_{1} \\
\vec{u}_{0}
\end{array}\right]=\left[\begin{array}{c}
\vec{u}_{n+1} \\
\vec{u}_{n}
\end{array}\right]
$$

The corners imply a condition of periodicity $\vec{u}_{0}=\vec{u}_{n}$ and $\vec{u}_{n+1}=\vec{u}_{1}$, that can be used to obtain the eigenvalue $\epsilon$ in alternative to diagonalization of $H$. By comparing eqs.(2) and (5) one arrives at the main point: to study the spectrum of $T(\epsilon)$, one must impose the generalized b.c. (1). We thus introduce an instrumental non-Hermitian matrix depending on a parameter $z\left(0 \leq \arg z \leq \frac{2 \pi}{n}\right)$

$$
H\left(z^{n}\right)=\left[\begin{array}{cccc}
A_{1} & B_{1} & & \frac{1}{z^{n}} B_{n}^{\dagger} \\
B_{1}^{\dagger} & \ddots & \ddots & \\
& \ddots & \ddots & B_{n-1} \\
z^{n} B_{n} & & B_{n-1}^{\dagger} & A_{n}
\end{array}\right]
$$

The matrix is Hermitian for Bloch b.c. $(|z|=1)$ but, for the purpose of studying the spectrum of $T(\epsilon)$, it will be considered for $z \in C_{0}$. The matrix can be brought by similarity to the balanced form $H_{b}(z)=Z^{-1} H\left(z^{n}\right) Z$,

$$
H_{b}(z)=\left[\begin{array}{cccc}
A_{1} & z B_{1} & & \frac{1}{z} B_{n}^{\dagger} \\
\frac{1}{z} B_{1}^{\dagger} & \ddots & \ddots & \\
& \ddots & \ddots & z B_{n-1} \\
z B_{n} & & \frac{1}{z} B_{n-1}^{\dagger} & A_{n}
\end{array}\right]
$$

by means of the block diagonal matrix $Z$ with blocks $\left\{z I_{m}, \ldots, z^{n} I_{m}\right\}$. Therefore, no site of the chain is privileged. While the matrix $H_{b}(z)$ does change if $\arg z$ is increased by $2 \pi / n$, its eigenvalues do not.

\section{B. Symplectic properties and exponents}

The following relations hold for the transfer matrix:

$$
\begin{aligned}
& T\left(\epsilon^{*}\right)^{\dagger} \Sigma_{n} T(\epsilon)=\Sigma_{n}, \quad \Sigma_{n}=\left[\begin{array}{cc}
0 & -B_{n}^{\dagger} \\
B_{n} & 0
\end{array}\right] \\
& T(\epsilon) \Sigma_{n}^{-1} T\left(\epsilon^{*}\right)^{\dagger}=\Sigma_{n}^{-1}, \quad \Sigma_{n}^{-1}=\left[\begin{array}{cc}
0 & B_{n}^{-1} \\
-B_{n}^{\dagger-1} & 0
\end{array}\right]
\end{aligned}
$$

Let us denote as $z_{1}^{n} \ldots z_{2 m}^{n}$ the $2 m$ eigenvalues of $T(\epsilon)$. The relations imply that if $z_{a}^{n}$ is an eigenvalue of $T(\epsilon)$, then $\left(z_{a}^{-n}\right)^{*}$ is an eigenvalue of $T\left(\epsilon^{*}\right)$. In this study we are concerned with the exponents

$$
\xi_{a}(\epsilon)=\log \left|z_{a}\right|
$$

In general they still depend on $n$. Since $|\operatorname{det} T(\epsilon)|=1$, it is always $\sum_{a} \xi_{a}(\epsilon)=0$. For real $\epsilon$ the exponents of $T(\epsilon)$ come in pairs $\pm \xi_{a}$.

\section{Duality, Jensen, and spectrum of exponents}

Since the extremal components $\vec{u}_{1}$ and $\vec{u}_{n}$ of the eigenvector $H\left(z^{n}\right) u=\epsilon u$ enter in the eigenvalue equation (2) of $T(\epsilon)$, it follows that the characteristic polynomials of the two matrices are linked by a

Duality relation. $\epsilon$ is an eigenvalue of $H\left(z^{n}\right)$ iff $z^{n}$ is eigenvalue of $T(\epsilon)$ :

$$
\frac{\operatorname{det}\left[\epsilon I_{n m}-H\left(z^{n}\right)\right]}{\operatorname{det}\left[B_{1} \cdots B_{n}\right]}=\frac{(-1)^{m}}{z^{n m}} \operatorname{det}\left[T(\epsilon)-z^{n} I_{2 m}\right]
$$

A proof of duality that holds also for non-Hermitian matrices, with blocks $B_{k}^{\dagger}$ being replaced by blocks $C_{k}$, is found in ref. $\stackrel{4}{*}$.

The spectrum of exponents can be obtained from the spectrum of $H\left(z^{n}\right)$ through the following identity for analytic functions, which is a particular case of a theorem by Poisson and Jensen for subharmonic functions ${ }^{10}$ :

Jensen's identity. Let $f$ be an analytic function in the open disk of radius $R$, where it has zeros $z_{1}, \ldots, z_{k}$ that are ordered according to increasing modulus. Then, if $0<\left|z_{1}\right|$ and for $r$ such that $\left|z_{\ell}\right| \leq r \leq\left|z_{\ell+1}\right|$ we have:

$$
\int_{0}^{2 \pi} \frac{d \varphi}{2 \pi} \log \left|f\left(r e^{i \varphi}\right)\right|=\log \frac{r^{\ell}|f(0)|}{\left|z_{1} \cdots z_{\ell}\right|}
$$

Proposition. For real $\xi$ and complex $\epsilon$ it is

$$
\begin{aligned}
& \frac{1}{m} \sum_{\xi_{a}<\xi}\left[\xi-\xi_{a}(\epsilon)\right]-\xi=-\frac{1}{n m} \sum_{k=1}^{n} \log \left|\operatorname{det} B_{k}\right| \\
& +\int_{0}^{2 \pi} \frac{d \varphi}{2 \pi} \frac{1}{n m} \log \left|\operatorname{det}\left[\epsilon I_{n m}-H\left(e^{n \xi+i \varphi}\right)\right]\right|
\end{aligned}
$$

Proof: Jensen's identity is applied to the polynomial $f(z)=\operatorname{det}\left[T(\epsilon)-z^{n} I_{2 m}\right]$, with $|f(0)|=1$ and $z=$ $e^{\xi+i \varphi / n}$. The duality relation is then used to obtain the formula.

For $\xi=0$ a formula for the sum of positive exponents follows. It involves a real eigenvalue spectrum

$$
\begin{aligned}
& \frac{1}{m} \sum_{\xi_{a}>0} \xi_{a}(\epsilon)=-\frac{1}{n m} \sum_{k} \log \left|\operatorname{det} B_{k}\right| \\
& +\int_{0}^{2 \pi} \frac{d \varphi}{2 \pi} \frac{1}{n m} \log \mid \operatorname{det}\left[\epsilon I_{n m}-H\left(e^{i \varphi}\right)\right] .
\end{aligned}
$$

Equations (12) and (13) are exact and valid for a single, general transfer matrix. In the theory of disordered systems, a formula for the sum of exponents is known, where Jensen's angular average is replaced by the ensemble average $11,12,13$.

The left-hand side of eq. (12) is a non decreasing function of $\xi$ (Fig, 1). For all $\xi \geq \xi_{M A X}(\epsilon)$ (the maximum 


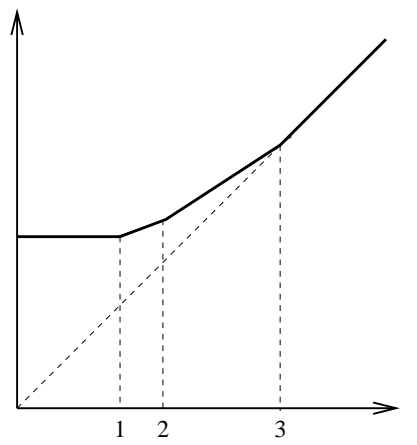

FIG. 1: The behaviour of the right hand side of eq. (12) as a function of $\xi$, for $m=3$. The constant value for $\xi<\xi_{1}=\xi_{\min }$ is $\frac{1}{3}\left(\xi_{1}+\xi_{2}+\xi_{3}\right)$. At $\xi=\xi_{1}, \xi_{2}$ and $\xi_{3}=\xi_{M A X}$ the slope increases by $1 / 3$.

exponent of $T(\epsilon)$ ), the right side is always equal to $\xi$. For all positive $\xi<\xi_{\text {min }}(\epsilon)$ (the smallest positive exponent), the right hand side is constant and equal to the average value of the exponents (13). For intermediate positive values of $\xi$ the function is piecewise linear, with discontinuities of order $1 / m$ in the first derivative, at the values of the exponents.

\section{The matrix $\mathbf{T}^{\dagger} \mathbf{T}$}

Let us introduce the matrix $Q(\epsilon)=T\left(\epsilon^{*}\right)^{\dagger} T(\epsilon)$, with exponents $\gamma_{a}(\epsilon)$. For real $\epsilon$ the matrix is real and positive, and is preferred to $T$ because of better large $n$ behaviour of the exponents. If $B_{n} B_{n}^{\dagger}=I_{m}$, the matrix $Q$ is symplectic

$$
Q(\epsilon) \Sigma_{n} Q(\epsilon)=\Sigma_{n}
$$

and the exponents come in pairs $\pm \gamma_{a}$. Hereafter, the matrix $B_{n}$ will be restricted to be unitary. Under this restriction, it is shown in Appendix A that $(-1)^{n} Q(\epsilon)$ is unitarily equivalent to the transfer matrix $\theta(\epsilon)$

$$
Q(\epsilon)=(-1)^{n}\left[\begin{array}{cc}
I_{m} & 0 \\
0 & B_{n}
\end{array}\right] \theta(\epsilon)\left[\begin{array}{cc}
I_{m} & 0 \\
0 & B_{n}^{\dagger}
\end{array}\right]
$$

of the block tridiagonal matrix $M(\epsilon)$ :

$$
\left[\begin{array}{cccccccc}
A_{1}-\epsilon & B_{1} & & & & & I_{m} \\
B_{1}^{\dagger} & \ddots & \ddots & & & & & \\
& \ddots & \ddots & B_{n-1} & & & & \\
& & B_{n-1}^{\dagger} & A_{n}-\epsilon & I_{m} & & & \\
& & & I_{m} & \epsilon-A_{n} & B_{n-1}^{\dagger} & & \\
& & & & B_{n-1} & \ddots & \ddots & \\
& & & & & \ddots & \ddots & B_{1}^{\dagger} \\
& & & & & & B_{1} & \epsilon-A_{1}
\end{array}\right]
$$

Factors $z^{ \pm 2 n}$ are then introduced in the corners as in eq.(3) and, because of the factor $(-1)^{n}$ in (15), the duality relation is as follows:

$$
\frac{\operatorname{det} M\left(\epsilon, z^{2 n}\right)}{\prod_{k}\left|\operatorname{det} B_{k}\right|^{2}}=\frac{(-1)^{m}}{z^{2 n m}} \operatorname{det}\left[Q(\epsilon)-(i z)^{2 n} I_{2 m}\right]
$$

Since only the determinant matters, there is freedom to modify $M$ to a form where $\epsilon$ enters as a shift. Left and right multiplication by the block diagonal matrices $\left\{I_{n m}, I_{m},-I_{m},+I_{m}, \ldots\right\}$ and $\left\{I_{n m},-I_{m}, I_{m},-I_{m}, \ldots\right\}$ give $\operatorname{det} M=(-1)^{n m} \operatorname{det}\left[K\left((i z)^{2 n}\right)-\epsilon I_{2 n m}\right]$, with $K\left((i z)^{2 n}\right)=$

$$
\left[\begin{array}{cccccccc}
A_{1} & B_{1} & & & & & & \frac{1}{(i z)^{2 n}} \\
B_{1}^{\dagger} & \ddots & \ddots & & & & & \\
& \ddots & \ddots & B_{n-1} & & & & \\
& & B_{n-1}^{\dagger} & A_{n} & -I_{m} & & & \\
& & & I_{m} & A_{n} & B_{n-1}^{\dagger} & & \\
& & & & B_{n-1} & \ddots & \ddots & \\
& & & & & \ddots & \ddots & B_{1}^{\dagger} \\
-(i z)^{2 n} & & & & & & B_{1} & A_{1}
\end{array}\right]
$$

A true duality relation among eigenvalues is obtained (replace $i z$ by $z$ )

$$
\frac{\operatorname{det}\left[K\left(z^{2 n}\right)-\epsilon I_{2 n m}\right]}{\prod_{k}\left|\operatorname{det} B_{k}\right|^{2}}=\frac{(-1)^{m}}{z^{2 n m}} \operatorname{det}\left[Q(\epsilon)-z^{2 n} I_{2 m}\right]
$$

The Lyapunov spectrum is extracted by means of Jensen's formula:

$$
\begin{aligned}
& \xi+\frac{1}{m} \sum_{\gamma_{a}>\xi}\left[\gamma_{a}(\epsilon)-\xi\right]=\frac{1}{n m} \sum_{k=1}^{n-1} \log \left|\operatorname{det} B_{k}\right| \\
& +\int_{0}^{2 \pi} \frac{d \varphi}{2 \pi} \frac{1}{2 n m} \log \left|\operatorname{det}\left[K\left(e^{2 n \xi+i \varphi}\right)-\epsilon I_{2 n m}\right]\right|
\end{aligned}
$$

Some properties of the matrix $K$ are presented in Appendix B.

\section{THE ANDERSON MODEL}

The discrete Anderson model describes a particle in a lattice, subject to a random potential. The potential of a sample is specified by a set $\left\{v_{\mathbf{j}}\right\}$ of random numbers chosen independently. Anderson ${ }^{14}$ considered a uniform density $p(v)=1 / w$ in the interval $[-w / 2, w / 2]$. Lloyd $^{15,16}$ studied the Cauchy distribution $p(v)=\frac{\delta}{\pi}\left(v^{2}+\right.$ $\left.\delta^{2}\right)^{-1}$, and evaluated the energy distribution exactly in any space dimension. Anderson's choice and the simple hypercubic geometry are here considered. More complex lattices can be studied by transfer matrix ${ }^{17}$.

For a given configuration of potential, the eigenvalue equation is

$$
\sum_{\mathbf{e}} u_{\mathbf{j}+\mathbf{e}}+v_{\mathbf{j}} u_{\mathbf{j}}=\epsilon u_{\mathbf{j}}
$$



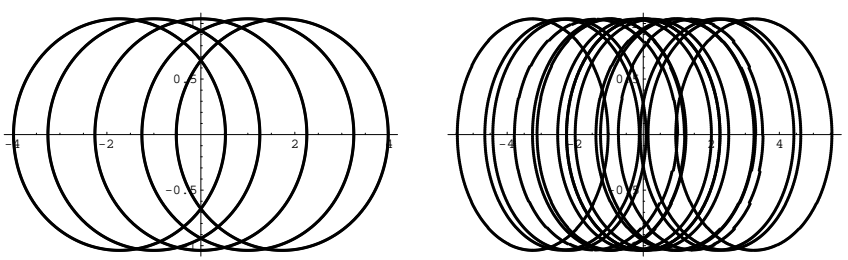

FIG. 2: Complex energy spectra for zero disorder, large $n$, $\xi=1$ : in $2 \mathrm{D}$ (left, $m=5$ ) and $3 \mathrm{D}$ (right, $n_{x}=n_{y}=4$ i.e. $m=16)$.

The sum is on the unit vectors along the $2 D$ directions, $\epsilon$ is the energy of the particle, the lattice has lengths $n_{1}, \ldots, n_{D}$. If the $D$ axis is singled out, the sample is viewed as a number $n \equiv n_{D}$ of sections each containing $m \equiv n_{1} \cdots n_{D-1}$ sites. Accordingly, the Hamiltonian matrix is block tridiagonal

$$
H=\left[\begin{array}{cccc}
A_{1} & I_{m} & & I_{m} \\
I_{m} & \ddots & \ddots & \\
& \ddots & \ddots & I_{m} \\
I_{m} & & I_{m} & A_{n}
\end{array}\right]
$$

with Hermitian blocks $A_{i}$ describing sections, and off diagonal blocks describing hopping among sections. The associated transfer matrix is

$$
T(\epsilon)=\prod_{j=1}^{n}\left[\begin{array}{cc}
\epsilon I_{m}-A_{j} & -I_{m} \\
I_{m} & 0
\end{array}\right]
$$

For large $n$ the exponents of $T(\epsilon)$ describe the inverse decay lengths of the eigenstates of Anderson's Hamiltonian. To study them, we introduce b.c. terms $\pm z^{n}$ in the corner blocks of (19), and choose periodic b.c. in the other $D-1$ directions, that appear in the diagonal blocks.

Remark 0: For zero disorder the eigenvalues of $H\left(z^{n}\right)$ are complex for any nonzero value of the parameter $\xi$ that measures non-Hermiticity $\left(z=e^{\xi+i \varphi}\right)$ :

$$
\begin{aligned}
& \operatorname{Re} \epsilon=2 \cosh \xi \cos \left(\varphi+\frac{2 \pi}{n} \ell\right)+\epsilon_{r} \\
& \operatorname{Im} \epsilon=2 \sinh \xi \sin \left(\varphi+\frac{2 \pi}{n} \ell\right),
\end{aligned}
$$

$\ell=1, \ldots, n$ and $r=1, \ldots, m$. There are $n$ eigenvalues on each ellipse centered at $\epsilon_{r}=2 \sum_{i=1}^{D-1} \cos \left(2 \pi \frac{k_{i}}{n_{i}}\right)$, $1 \leq k_{i} \leq n_{i}$. Therefore, the spectrum has support on $m$ identical but shifted ellipses. In $1 D$ there is a single ellipse centered in the origin. In $2 D$ there are $m=n_{x}$ distinct ones, while in $3 D$ some of the $m=n_{x} n_{y}$ ellipses may overlap because centers may be degenerate (Fig 2). In Appendix $\mathrm{C}$ it is shown that the exponents of $T$ and $T^{\dagger} T$ coincide, for large $n$.

Remark 1: For non-zero disorder the eigenvalues of the Hamiltonian matrix $H\left(z^{n}\right)$ are all contained inside the union of ellipses

$$
\frac{\left(\operatorname{Re} \epsilon-\epsilon_{0}\right)^{2}}{4 \cosh ^{2} \xi}+\frac{(\operatorname{Im} \epsilon)^{2}}{4 \sinh ^{2} \xi} \leq 1
$$

where $\epsilon_{0}$ ranges in the interval $[-2 D+2-w / 2,2 D-2+$ $w / 2$. Proof: If $H_{b}(z) u=\epsilon u$, and $u$ is normalized, the inner product $\epsilon=\left(u \mid H_{b}(z) u\right)$ in $C^{n m}$ is separated into real and imaginary parts:

$$
\begin{aligned}
& \operatorname{Re} \epsilon-(u \mid A u)=2|(u \mid S u)| \cosh \xi \cos (\varphi+\theta) \\
& \operatorname{Im} \epsilon=2|(u \mid S u)| \sinh \xi \sin (\varphi+\theta)
\end{aligned}
$$

$A$ is the block diagonal part of $H_{b}, S$ is the one-block shift matrix, and $\theta=\arg (u \mid S u)$. The real number $(u \mid A u)=\epsilon_{0}$ ranges in the spectrum of $A$. Schwartz's inequality gives the bounds.

Remark 2: Since $H$ is real, under complex conjugation it is $T(\epsilon)^{*}=T\left(\epsilon^{*}\right)$. Then $\xi_{a}(\epsilon)=\xi_{a}\left(\epsilon^{*}\right)$. The symplectic property (8) with $B_{n}=I_{m}$ implies that the exponents of $T(\epsilon)$ come in pairs $\pm \xi_{a}$ for any $\epsilon$.

Remark 3: Since the transposed matrix $H\left(z^{n}\right)^{t}$ coincides with $H\left(z^{-n}\right)$, then $\operatorname{det}\left[\epsilon I_{n m}-H\left(z^{n}\right)\right]$ is a polynomial of degree $m$ of the variable $\left(z^{n}+z^{-n}\right)$.

Remark 4: Since $H_{b}\left(z e^{i 2 \pi / n}\right) \simeq H_{b}(z)(\simeq$ means similarity) and $H_{b}(z)^{*}=H_{b}\left(z^{*}\right)$, the following symmetry holds: $H_{b}\left(e^{\xi+i\left(\frac{2 \pi}{n}-\varphi\right)}\right) \simeq H_{b}\left(e^{\xi+i \varphi}\right)^{*}$.

\section{A. The Lyapunov spectrum}

The localization properties of Anderson's model are usually derived from the spectrum of positive Lyapunov exponents $\gamma_{1}<\ldots<\gamma_{m}$ of $T(\epsilon)^{\dagger} T(\epsilon)$, with $\epsilon$ real. Oseledec's theorem 18 guarantees that for large $n$ it does not depend on the length $n$, and on the realization of disorder. The most interesting exponent for physics is $\gamma_{1}$, that controls conductance. It is also the most difficult one to study numerically, because of the larger ones ${ }^{19,20}$. Thorough investigations of the Lyapunov spectrum, its statistical properties and scaling, have been done in $2 \mathrm{D}^{21}$ and $3 \mathrm{D}^{22}$. The influence of b.c. was studied 23 with the corner parameters $z^{ \pm n}$ of the present theory being both replaced by the same parameter $t \in[0,1]$. It was found that the critical values of $\gamma_{1}$ and of the disorder parameter $w_{c}$ are $t$-dependent, while the critical exponent $\nu$ is not.

Analytic results for the Lyapunov spectrum are accessible in perturbation theory for the 2D strip $24,25,26$, where $n$ is large and $m$ is finite. In such quasi-1D Anderson systems, the large $n$ limit of the exponent spectrum of $T$ coincides with the Lyapunov spectrum. For this reason here I concentrate on the spectral features of the matrix $H\left(z^{n}\right)$ to which $T$ is linked by duality. I'll then show in another section that the spectral identity (12) allows to evaluate the smallest exponent $\xi_{\min }$, which converges to $\gamma_{1}$ for large $n$.

\section{B. 1D Anderson model, Hatano and Nelson.}

For a chain of $n$ sites the Hamiltonian is a tridiagonal $n \times n$ matrix and the transfer matrix is $2 \times 2$. Hatano 

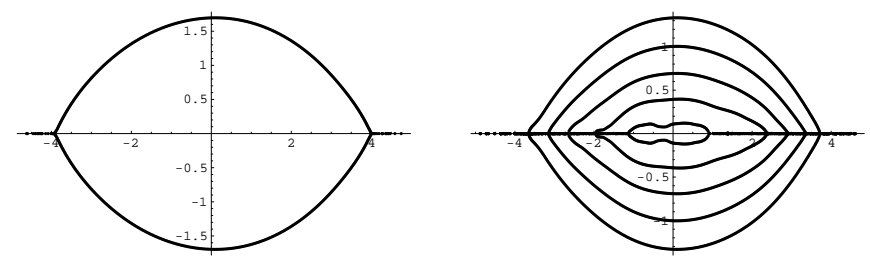

FIG. 3: 1D Anderson model: Left: the complex eigenvalues form a closed loop, with two wings of real values $(w=7$, $n=600, \xi=1)$. Right: superposition of the eigenvalues of five matrices (same $w$ and $n$ ) for $\xi$ from 0.5 (inner blob) to 1 (outer).

and Nelson ${ }^{\frac{8}{}}$ suggested to study 1D Anderson localization through the non-Hermitian extension of the model

$$
e^{\xi} \psi_{i+1}+e^{-\xi} \psi_{i-1}+v_{i} \psi_{i}=\epsilon \psi_{i}
$$

with periodic b.c. As $\xi$ is increased from zero, the eigenvalues do not distribute randomly in the complex plane but form a loop, Fig 3 (left), whose analytic expression is known for Cauchy disorder ${ }^{28}$. The loop has two outer wings of real eigenvalues, that correspond to enough localized eigenstates, and evolves to a more and more regular shape, while the wings reduce, Fig 3 (right). The value $\xi_{c}(\epsilon)$ up to which an eigenvalue $\epsilon$ persists in the real axis, measures the inverse localization length of the physical eigenvector (the Lyapunov exponent): $\xi_{c}=\gamma_{1}(\epsilon)$. The latter is evaluated through Herbert, Jones and Thouless' formula,

$$
\gamma_{1}(\epsilon)=\int d \epsilon^{\prime} \rho\left(\epsilon^{\prime}\right) \log \left|\epsilon-\epsilon^{\prime}\right|
$$

where $\rho(\epsilon)$ is the disorder-averaged level density of the matrix ensemble in the limit of large $n, \xi=0$. The model has been studied by several authors $30,31,32,33,34$; mathematical proofs were established by Goldsheid and Khoruzhenko ${ }^{35}$. Diagonalization of large non-Hermitian matrices is a delicate issue, as approximate eigenvalues may occur which are not close to true ones ${ }^{36}$.

The Hatano-Nelson model is a case $m=1$ of the theory presented in Section II. The duality relation (10) simplifies greatly:

$$
\operatorname{det}\left[\epsilon I_{n}-H\left(z^{n}\right)\right]=\operatorname{tr} T(\epsilon)-\left(z^{n}+z^{-n}\right)
$$

and implies that

$$
\operatorname{tr} T(\epsilon)=\operatorname{det}\left[\epsilon I_{n}-H(i)\right] \equiv p_{n}(\epsilon)
$$

For a pure Bloch phase eq.(24) describes the energy bands of $H\left(e^{i n \varphi}\right)$ as intersections of the polynomial $y=$ $p_{n}(\epsilon)$ with the strip $y=2 \cos (n \varphi)$. As the non-Hermitian regime is entered, $y= \pm 2 \cosh (n \xi)$, all eigenvalues of $H\left(e^{n \xi}\right)$ are in the gaps, and approach pairwise for increasing $\xi$. A pair collides at a zero of $p_{n}^{\prime}$ and becomes complex conjugate. This means that $2 \cosh (n \xi)$ equals the height $\left|p_{n}\right|$ at an extremum of the polynomial.

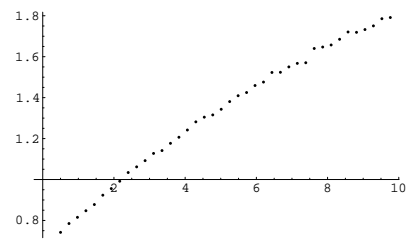

FIG. 4: 1D Anderson model: the exponent $\xi_{1}(0)$ versus disorder parameter $w$, for $n=600$, averaged over 8 samples of disorder.

The sum of the two eigenvalues $e^{ \pm n\left(\xi_{1}+i \varphi_{1}\right)}$ of the transfer matrix is

$$
p_{n}(\epsilon)=\cosh \left(n \xi_{1}\right) \cos \left(n \varphi_{1}\right)+2 i \sinh \left(n \xi_{1}\right) \sin \left(n \varphi_{1}\right)
$$

Elimination of the phase results in an exact equation for the exponent $\xi_{1}(\epsilon)$

$$
\frac{\left(\operatorname{Re} p_{n}\right)^{2}}{4 \cosh ^{2}\left(n \xi_{1}\right)}+\frac{\left(\operatorname{Im} p_{n}\right)^{2}}{4 \sinh ^{2}\left(n \xi_{1}\right)}=1
$$

For large $n$ it becomes $e^{n \xi_{1}(\epsilon)}=\left|p_{n}(\epsilon)\right|$, and gives a convenient formula to compute the exponent (Fig (4)

$$
\xi_{1}(\epsilon)=\frac{1}{n} \log \left|\operatorname{det}\left[\epsilon I_{n}-H(i)\right]\right|
$$

In $1 \mathrm{D}$ the single exponent is also given by the exact formula eq.(13). For large $n, \xi_{1}$ coincides with the Lyapunov exponent $\gamma_{1}$.

Proposition: the eigenvalues of $H\left(e^{n \xi}\right)$ distribute along the curve $\xi_{1}(\epsilon)=\xi$. Real eigenvalues (wings) solve $p_{n}(\epsilon)=2 \cosh (n \xi)$. For large $n \xi$ the eigenvalues form the Lemniscate $^{27}\left|p_{n}(\epsilon)\right|=e^{n \xi}$.

\section{2D Anderson model}

For a rectangular $n \times m$ lattice the Hamiltonian matrix (19) has diagonal blocks

$$
A_{i}=\left[\begin{array}{cccc}
v_{i, 1} & 1 & & 1 \\
1 & \ddots & \ddots & \\
& \ddots & \ddots & 1 \\
1 & & 1 & v_{i, m}
\end{array}\right]
$$

The eigenvalue spectrum of 2D non-Hermitian Anderson model is studied, and explained in the light of duality. Fig $\left[5\right.$ shows the eigenvalues of two matrices $H\left(e^{n \xi}\right)$ with same $\xi$ and different $m$. They are distributed along a number of loops which is precisely given by $m$, the size of the blocks.

By varying only the phase $\varphi$ of $z$, the eigenvalues of the matrix $H_{b}(z)$ move in the complex plane along arcs which retrace the loops. Fig 6 shows that, as $\varphi$ goes from 0 to $2 \pi / n$, an eigenvalue moves along an arc that 

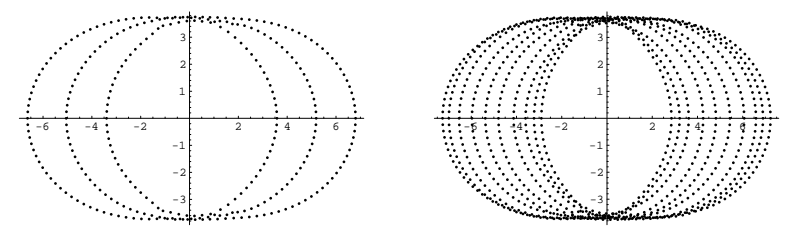

FIG. 5: 2D Anderson model: eigenvalues of a single matrix, with parameters $w=7, n=100, \xi=1.5, \varphi=0$. Size of blocks: $m=3$ and $m=10$. The size $m$ of blocks is the number of loops.
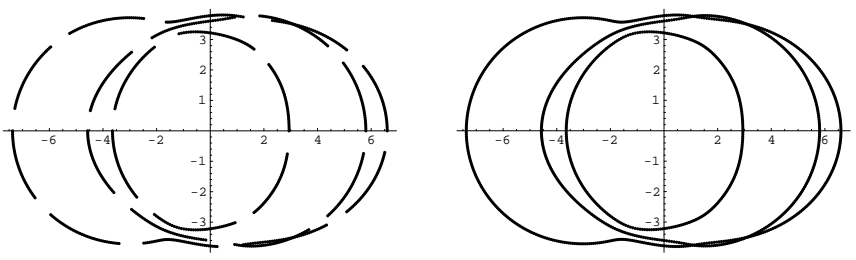

FIG. 6: 2D Anderson model: motion of the eigenvalues in the complex plane for fixed disorder $\mathrm{w}=7$ and parameters $m=3$, $n=8$, and $\xi=1.5$ and varying the phase $\varphi$. The 24 eigenvalues trace arcs: $0 \leq \varphi \leq \pi / 4-\delta$ (left) and $0 \leq \varphi \leq \pi / 4$ (right). The arcs join to form three loops. Loops are seen to contain different numbers of eigenvalues.

terminates where the arc of another eigenvalue starts. The union of such consecutive arcs makes a loop, and there are $m$ closed loops. Differently from the $w=0$ case, loops may contain different numbers of eigenvalues of the matrix. The occurrence of loops is suggested by the duality equation: when a zero of $\operatorname{det}\left[H_{b}(z)-\epsilon I_{n m}\right]$ occurs, it is also a zero of $\operatorname{det}\left[T(\epsilon)-z^{n}\right]$, or

$$
\xi_{a}(\epsilon)=\xi, \quad \varphi_{a}(\epsilon)=\varphi \quad \bmod \frac{2 \pi}{n}, \quad(a=1, \ldots, m)
$$

The loops are thus level curves of the exponents $\xi_{a}$, as functions of the complex variable $\epsilon$. In the limit of large $n$, the eigenvalues of one matrix fill $m$ loops.

In Fig 7 only $\xi$ is varied: the eigenvalues trace lines that originate on the real axis (at $\xi=0$ the matrix is Hermitian). For zero disorder the lines would be arcs of hyperbola. In the disordered case, for small $\xi$ the pattern of eigenvalues is complex, but evolves to regular loops. In the next section it is shown that the evaluation of exponents requires a "regular" regime. In Fig 8 the parameters $\xi$ and $\varphi$ are kept fixed, and the eigenvalues are computed for different realizations of disorder, with same strength $w$. They distribute along $m$ loops, that appear shifted along the real axis for the different samples.

\section{The smallest exponent}

Let us assume that the exponents $\pm \xi_{a}$ of $T(\epsilon)$ are isolated, $0<\xi_{\min }<\xi_{2} \ldots<\xi_{m-1}<\xi_{M A X}$. By increasing

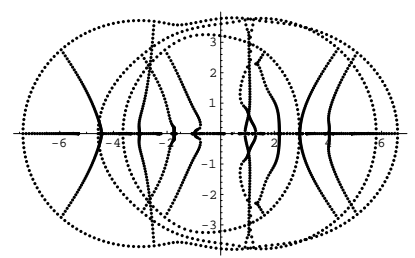

FIG. 7: 2D Anderson model. Motion of 24 eigenvalues for $0 \leq \xi \leq 1.5, w=7, m=3, n=8, \varphi=0$. The various wings terminate on $m=3$ loops (at $\xi=1.5$ the phase is allowed to vary over $2 \pi$.)
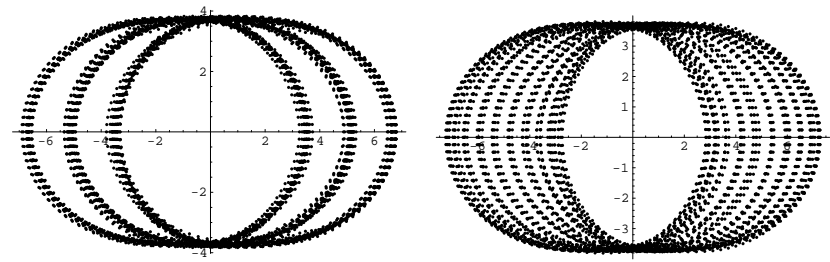

FIG. 8: 2D Anderson model: superposition of eigenvalue spectra for various realizations of disorder. For all: $\mathrm{w}=7, n=100$, $\xi=1.5, \varphi=0$. Left: $m=3,20$ realizations of disorder. Right: $m=10,5$ realizations. The imaginary part of the eigenvalues is much less sensitive to disorder sampling than the real part.

$\xi$ from the value zero, the r.h.s. of eq. (12) yields a constant value (the $\xi=0$ value) until the value $\xi=\xi_{\min }(\epsilon)$ is reached. Then the function becomes linear with slope $1 / m$ until the value $\xi_{2}(\epsilon)$ is reached, where a new change of slope occurs. The change of slope can be used to identify the smallest exponent. Fig 9 illustrates this behaviour. Fig 10 shows that for $\xi \approx \xi_{\min }$ the eigenvalues of the matrix are all well in the complex plane.

Kuwae and Taniguchi ${ }^{37}$ extended Hatano Nelson's approach to $2 \mathrm{D}$ Anderson model, and evaluated numerically the average critical value $\xi_{c}(w)$ where the first pair of eigenvalues turns to complex. They conjectured that the inverse of the localization length coincides with this critical value, i.e. $\gamma_{1}=\xi_{c}$. For example, for $m=n=20, w=7$ they evaluate $\xi_{c} \approx 0.1$. Fig 9 shows that $\xi_{\text {min }} \approx 0.446$, a measure of the inverse localization length. For this $\xi$ the spectrum is evaluated and found in the complex plane. These facts adverse Kuwae's hypothesis that $\gamma_{1}$ signals the first escape to complex of the eigenvalues of Anderson's non-Hermitian Hamiltonian, and require further study.

\section{CONCLUSIONS}

Based on a spectral duality relation for block tridiagonal matrices and Jensen's identity, the distribution of exponents of a transfer matrix can be evaluated from the eigenvalue spectrum of the Hamiltonian with nonHermitian boundary conditions. A preliminary numerical study of the complex energy spectra of Anderson 


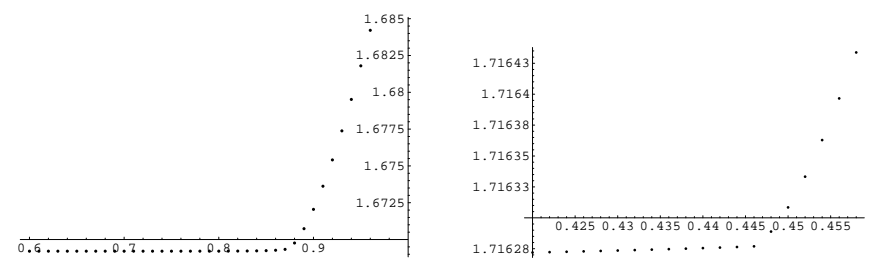

FIG. 9: 2D Anderson model: evaluation of the r.h.s. of eq. (12) as a function of $\xi<1$, for $\epsilon=0, w=7$, average on 40 angles. The change of slope marks $\xi_{\min }(0)$. Left: $m=3, n=50, \xi_{\min }(0) \approx 0.87$; the value 1.6692 is the average $\frac{1}{3}\left(\xi_{1}+\xi_{2}+\xi_{3}\right)$. Right: $m=n=20, \xi_{\min }(0) \approx 0.447$. The value 1.71627 is the average $\frac{1}{20}\left(\xi_{1}+\ldots+\xi_{20}\right)$.

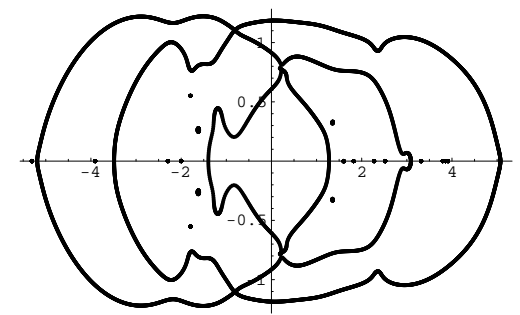

FIG. 10: 2D Anderson model: the eigenvalues for $\xi=0.835$, $\epsilon=0, w=7, m=3, n=50$. For $\xi$ near $\xi_{\text {min }} \approx 0.87$ the inner loop is isolated.

non-Hermitian Hamiltonian matrices is made. The spectra have support on loops, that are explained as sections of the exponents at fixed height: $\xi_{a}(\epsilon)=\xi$. This picture is complementary to a standard direct evaluation of the exponents $\xi_{a}(\epsilon)$ at fixed energy $\epsilon$, by diagonalization of the transfer matrix.

The spectral formula for exponents allows to evaluate the smallest one, and involves eigenvalues of the nonHermitian Hamiltonian that are away from the real axis and distributed in loops that are nearly untertwined. This, and the higher exponents, are interesting subjects for further investigation.

\section{Appendix A}

We show the relationship of $Q(\epsilon)=T\left(\epsilon^{*}\right)^{\dagger} T(\epsilon)$ with a transfer matrix. It is convenient to factor $T(\epsilon)$ as

$$
\left[\begin{array}{cc}
B_{n}^{-1} & 0 \\
0 & I_{m}
\end{array}\right] t_{n} \sigma_{n-1} \cdots \sigma_{1} t_{1}\left[\begin{array}{cc}
I_{m} & 0 \\
0 & B_{n}^{\dagger}
\end{array}\right]
$$

Accordingly:

$$
\begin{gathered}
Q(\epsilon)=(-1)^{n}\left[\begin{array}{cc}
I_{m} & 0 \\
0 & B_{n}
\end{array}\right] u_{1} \sigma_{1}^{\dagger} \cdots \sigma_{n-1}^{\dagger} u_{n} \\
\times\left[\begin{array}{cc}
\left(B_{n} B_{n}^{\dagger}\right)^{-1} & 0 \\
0 & I_{m}
\end{array}\right] t_{n} \sigma_{n-1} \cdots \sigma_{1} t_{1}\left[\begin{array}{cc}
I_{m} & 0 \\
0 & B_{n}^{\dagger}
\end{array}\right] \\
t_{k}=\left[\begin{array}{cc}
\epsilon I_{m}-A_{k} & -I_{m} \\
I_{m} & 0
\end{array}\right], \quad u_{k}=\left[\begin{array}{cc}
A_{k}-\epsilon I_{m} & -I_{m} \\
I_{m} & 0
\end{array}\right], \\
\sigma_{k}=\left[\begin{array}{cc}
B_{k}^{-1} & 0 \\
0 & B_{k}^{\dagger}
\end{array}\right]
\end{gathered}
$$

To obtain the structure (29) of a transfer matrix, it is necessary that $B_{n}^{\dagger} B_{n}=I_{m}$. The first and last factors containing $B_{n}$ are not consistent with (29), and only the intermediate product $u_{1} \sigma_{1}^{\dagger} \cdots \sigma_{1} t_{1}$ is the transfer matrix of a tridiagonal block matrix, eq.(15).

Appendix B

One can show the following properties of the matrix $K(s) . J$ is the matrix with $2 n$ blocks $I_{m}$ along the diagonal from lower left to upper right corners; $S_{3}$ is the block diagonal matrix $\left\{I_{n m},-I_{n m}\right\}$. Then:

$$
\begin{array}{r}
J K(s) J=K\left(s^{*}\right)^{\dagger} \\
S_{3} K(s) S_{3}=K\left(1 / s^{*}\right)
\end{array}
$$

The two imply that $K(s)$ is similar to $K(1 / s)$.

\section{Appendix C}

For the Anderson model with no disorder $(w=0)$, real energy $\epsilon$ and large $n$, the exponents of $T$ and $T^{\dagger} T$ coincide. Proof: It is

$$
\begin{aligned}
& T=\left[\begin{array}{cc}
\epsilon I_{m}-A & -I_{m} \\
I_{m} & 0
\end{array}\right]^{n} \\
& =\left[\begin{array}{cc}
U & 0 \\
0 & U
\end{array}\right]\left[\begin{array}{cc}
\epsilon I_{m}-\Lambda & -I_{m} \\
I_{m} & 0
\end{array}\right]^{n}\left[\begin{array}{cc}
U^{\dagger} & 0 \\
0 & U^{\dagger}
\end{array}\right]
\end{aligned}
$$

where $A=U \Lambda U^{\dagger}$ and $\Lambda$ is the diagonal matrix of eigenvalues $\left\{\lambda_{1}, \ldots \lambda_{m}\right\}$. The eigenvalues of $T$ are $m$ pairs $z_{k}^{ \pm n}$, where $z_{k}$ is a root of the equation $z_{k}^{2}-\left(\epsilon-\lambda_{k}\right) z_{k}+1=$ 0 .

The power $n$ of the matrix can be computed by means of Cayley-Hamilton's formula. Because the blocks are diagonal, only powers zero and one of the matrix are needed:

$$
\left[\begin{array}{cc}
\epsilon I_{m}-\Lambda & -I_{m} \\
I_{m} & 0
\end{array}\right]^{n}=\left[\begin{array}{cc}
\alpha & 0 \\
0 & \alpha
\end{array}\right]+\left[\begin{array}{ll}
\beta & 0 \\
0 & \beta
\end{array}\right]\left[\begin{array}{cc}
\epsilon I_{m}-\Lambda & -I_{m} \\
I_{m} & 0
\end{array}\right]
$$

$\alpha$ and $\beta$ are diagonal matrices with elements constructed with the roots $z_{k}: z_{k}^{ \pm n}=\alpha_{k}+\beta_{k} z_{k}^{ \pm 1}$. Since $\epsilon$ is real, $\alpha_{k}$ and $\beta_{k}$ are real. The matrix $T^{\dagger} T$ is then constructed, and diagonalized. Its eigenvalues are pairs $w_{k}^{ \pm 1}$, with sum

$$
w_{k}+w_{k}^{-1}=\left(z_{k}^{2 n}+z_{k}^{-2 n}\right)\left(\frac{z_{k}^{2}+1}{z_{k}^{2}-1}\right)^{2}-\frac{8 z_{k}^{2}}{\left(z_{k}^{2}-1\right)^{2}}
$$

If $\left|z_{k}\right|>1$ then, for large $n,\left|w_{k}\right| \approx\left|z_{k}\right|^{2 n}$ (the spectrum of Lyapunov exponents of $T^{\dagger} T$ and the spectrum of exponents of $T$ coincide)

Acknowledgements I wish to thank professors Leonid Pastur, Borys Khoruzhenko, and especially Ilya Goldsheid for their interesting suggestions, while at the meeting "Anderson Localization and Related Phenomena" (aug 2008, Newton Institute, Cambridge). 
${ }^{1}$ L. Molinari, Transfer matrices and tridiagonal-block Hamiltonians with periodic and scattering boundary conditions, J. Phys. A: Math. Gen. 30 (1997) 983-997.

2 L. Molinari, Transfer matrices, non-Hermitian Hamiltonians and resolvents: some spectral identities, J. Phys. A: Math. Gen. 31 (1998) 8553-8562.

3 L. Molinari, Spectral duality and distribution of exponents for transfer matrices of block-tridiagonal Hamiltonians, J. Phys. A: Math. Gen. 36 (2003) 4081-4090.

4 L. G. Molinari, Determinants of block-tridiagonal matrices, Linear Algebra and its Applications 429 (2008), 22212226; also available at arXiv:0712.0681 [math-ph].

5 R. Nabben, Decay rates of the inverse of nonsymmetric tridiagonal and band matrices, Siam J. Matrix Anal. Appl. 20 (1999) 820-837.

6 E. Korotyaev and A. Kutsenko, Lyapunov functions for periodic matrix-valued Jacobi operators, arXiv:math/0701448 [math.SP].

7 H. Schulz-Baldes, Geometry of Weyl theory for Jacobi matrices with matrix entries, arXiv:0804.3746] [math-ph].

8 N. Hatano and D. R. Nelson, Localization transition in quantum mechanics, Phys. Rev. Lett. 77 (1996) 570.

9 J. Feinberg and A. Zee, Non-hermitian random matrix theory: method of hermitian reduction, Nucl. Phys. B 504 (1997) 579-608.

10 A. I. Markushevich, Theory of functions of complex variable, Chelsea Publishing Co. N.Y., 2nd Ed. (1977).

11 J. Lacroix, Computation of the sum of positive Lyapunov exponents for the Lloyd model on a strip, in "Lyapunov Exponents", Lect. Notes in Mathematics 1186 (1986).

12 L. Pastur and A. Figotin, Spectra of Random and AlmostPeriodic Operators, Grundlehren der mathematischen Wissenschaften 297, Springer-Verlag (1992).

13 B. Derrida, J. L. Jacobsen and R. Zeitak, Lyapunov exponent and density of states of a one-dimensional nonHermitian Schrödinger equation, J. Stat. Phys. 98 (2000) 31-55.

14 P. W. Anderson, Absence of diffusion in certain random lattices, Phys. Rev. 109 (1958) 1492.

15 P. Lloyd, Exactly solvable model of electronic states in a three-dimensional disordered Hamiltonian: non existence of localized states, J. Phys. C 2 (1969) 1717.

16 C. Mudry, P. W. Brouwer, B. I. Halperin, V. Gurarie and A. Zee, Density of states of non-hermitian Lloyd model, Phys. Rev. B 58 (1998) 13539.

17 A. Eilmes, A. M. Fisher and R. A. Römer, Critical parameters for the disorder-induced metal-insulator transition in FCC and BCC lattices, arXiv:0801.3338 [cond-mat.dis-nn].

18 A. Crisanti, G. Paladin, A. Vulpiani, Products of random matrices in statistical physics, Springer Series in solid state sciences 104, Springer-Verlag (1993).

19 J. L. Pichard and G. Sarma, Finite size scaling approach to Anderson localization, J. Phys. C: Solid State Phys. 14
(1981) L127.

20 A. MacKinnon and B. Kramer, One-parameter scaling of localization length and conductance in disordered systems Phys. Rev. Lett. 47 (1981) 1546.

${ }^{21}$ K. Slevin, Y. Asada and L. I. Deych, Fluctuations of the Lyapunov exponent in a two-dimensional disordered system, Phys. Rev. B 70 (2004) 054201.

22 P. Markoŝ, Universal scaling of Lyapunov exponents, J. Phys. A: Math. Gen. 30 (1997) 3441.

${ }^{23}$ V. Z. Cerovski, Boundary hopping and the mobility edge in the Anderson model in three dimensions, Phys. Rev. B 75 (2007) 113101.

24 Y. Rutman, M. Feingold, Y. Avishai and O. Piro, Localization in quasi-1D systems: perturbation theory and scaling, Europhys. Lett. 28 (1994) 329.

${ }^{25}$ Y. Rutman, M. Feingold and Y. Avishai, Localization in quasi-one-dimensional systems with random magnetic field, Phys. Rev. B 53 (1996) 9634.

26 R. A. Römer and H. Schulz-Baldes, Weak-disorder expansion for localization lengths of quasi1D systems, Europhys. Lett. 68 (2004) 247.

27 E. Hille, Analytic Function Theory, 2nd Ed. Chelsea, New York (1982).

28 I. Goldsheid and B. Khoruzhenko, Distribution of eigenvalues in non-Hermitian Anderson models, Phys. Rev. Lett. 80 (1998) 2897.

29 A. V. Kolesnikov and K. B. Efetov, Localization - delocalization transition in non-Hermitian disordered systems, Phys. Rev. Lett. 84 (2000) 5600.

${ }^{30}$ P. W. Brouwer, P. G. Silvestrov and C. W. J. Beenakker, Theory of directed localization in one dimension, Phys. Rev. B 56 (1997) R4333 - R4335.

31 N. M. Shnerb and D. R. Nelson, Winding numbers, complex currents, and non-Hermitian localization, Phys. Rev. Lett. 80 (1998) 5172.

32 J. Feinberg and A. Zee, Non-Hermitian localization and delocalization, Phys. Rev. E 59 (1999) 6433.

33 R. A. Janik, M. A. Nowak, G. Papp and I. Zahed, Localization transition from Free Random Variables, Acta Phys. Polonica B30 (1999) 45.

34 J. Heinrichs, Eigenvalues in the non-Hermitian Anderson model, Phys. Rev. B 63 (2001) 165108.

35 I. Y. Goldsheid and B. Khoruzhenko, Regular spacings of complex eigenvalues in the one-dimensional non-Hermitian Anderson model, Comm. Math. Phys. 238 (2003) 505-524.

36 E. B. Davies, Spectral properties of random non-selfadjoint matrices and operators, Proc. R. Soc. Lond. A 457 (2001) 191-206.

37 T. Kuwae and N. Taniguchi, Two dimensional nonHermitian delocalization transition as a probe for the localization length, Phys. Rev. B 64, 202321(R) (2001). 